Patrícia Andréa Rodrigues FerReria ${ }^{1}$

Luis Felipe Trincas Assad Sallum

LUIS OTÁ́vio SARIAN'

LIIANa A. LuGCi De Angelo ANDRADE ${ }^{2}$

SOPHIE DERCHAIN

Artigo Original

Palavras-chave

Neoplasias ovarianas/classificação Neoplasias ovarianas/patologia Neoplasias por tipo histológico

Carcinoma/classificação

Gradação de tumores Taxa de sobrevida

Prognóstico

Keywords

Ovarian neoplasms/classification Ovarian neoplasms/pathology Neoplasms by histologic type Carcinoma/classification Neoplasms grading Survival rate Prognosis

Sophie Derchain

Departamento de Ginecologia e Obstetricia da Faculdade de Ciênncios Médicas Universidade Estadual de Campinas - Caixa postal 611

CEP: $13083-970$

Campinas (SP), Brasi

Recebido

08/03/2012

Aceito com modificacōos

09/04/2012

\section{Carcinoma de ovário seroso e não seroso: tipo histológico em relação ao grau de diferenciação e prognóstico}

\author{
Serous and non-serous ovarian carcinoma: histological tumor type as \\ related to the grade of differentiation and disease prognosis
}

Resumo

OBJETIVO: Comparar as características clinicopatológicas de mulheres com carcinoma seroso e não seroso de ovário e identificar os fatores associados à sobrevida. MÉTODOS: Foram incluídas, neste estudo de coorte reconstituída, 152 mulheres com carcinoma de ovário, atendidas entre 1993 e 2008 e seguidas até 2010, nas quais o tipo histológico foi claramente estabelecido: 81 pacientes com carcinoma seroso e 71 pacientes com tumores não serosos 117 com carcinoma endometrioide, 44 com carcinoma mucinoso e 10 com carcinoma de células claras). Foram calculados os odds ratios (OR) brutos e os OR ajustados com os respectivos intervalos de confiança (IC95\%) para as características clínicas e patológicas, comparando tumores serosos e não serosos. Foram calculados os Hazard Ratios (HR) com os respectivos IC95\% em relação à sobrevida geral, para as variáveis clínicas e patológicas. RESULTADOS: Comparando os tipos seroso e não seroso, na análise univariada, os tumores serosos foram mais frequentes na pós-menopausa e eram preponderantemente carcinomas de alto grau histológico (G2 e G3), em estádios avançados, com CA 1 25>250 $\mathrm{U} / \mathrm{mL}$ e citologia peritoneal positiva. Após regressão múltipla, apenas o alto grau histológico se manteve associado com tumores serosos (OR ajustado 15, 1; IC95\% 2,9-77,9). Observamos 58 óbitos pela doença. O tipo histológico (seroso ou não seroso) não esteve associado com a sobrevida (HR 0,4; IC95\% 0,1-1, 1). Mulheres com idade de 50 anos ou menos (HR 0,4; IC95\% 0, 1-0,9) e aquelas que estavam em menacme (HR 0,3; IC95\% 0, 1-0,9) tiveram maior sobrevida quando comparadas, respectivamente, àquelas com idade acima de 50 anos e na menopausa. Carcinomas de alto grau histológico (G2 e $G 3)$ ( $p<0,01)$, estádio II a IV (p<0,008) e citologia peritoneal positiva $(p<0,001)$ estiveram significativamente relacionados com pior prognóstico. $\bigcirc$ nível sérico de CA 125 e a presença de ascite não se relacionaram com a sobrevida. A sobrevida foi menor quando a doença foi diagnosticada em estágios II a IV em comparação àquela das mulheres diagnosticadas no estádio I (log-rank $p<0,01$ ) independentemente do tipo histológico (seroso ou não seroso). CONCLUSÕES: A proporção de carcinomas de alto grau histológico (G2 ou G3) foi significativamente maior entre os carcinomas serosos comparados com não serosos. $\bigcirc$ tipo histológico seroso ou não seroso não esteve associado à sobrevida total.

\section{Abstract}

PURPOSE: To compare the clinical-pathological features of women with serous and non-serous ovarian tumors and to identify the factors associated with survival. METHODS: In this reconstructed cohort study, 152 women with ovarian carcinoma, who attended medical consultations between 1993 and 2008 and who were followed-up until 2010 were included. The histological type was clearly established for all women: 81 serous carcinomas and 71 non-serous tumors 117 endometrioid, 44 mucinous and 10 clear cell carcinomas). The crude and adjusted odds ratios (OR), with the respective $95 \%$ confidence intervals $(95 \% \mathrm{Cl})$, were calculated for the clinical and pathological features, comparing serous and non-serous histological types. The Hazard Ratios (HR) with $95 \% \mathrm{Cl}$ was calculated for overall survival, considering the clinical and pathological features. RESULTS: Comparison of serous to non-serous tumor types by univariate analysis revealed that serous tumors were more frequently found in postmenopausal women, and were predominantly high histological grade (G2 and G3), advanced stage, with CA125>250 U/mL, and with positive peritoneal cytology.

Trabalho realizado na Faculdade de Ciências Médicas da Universidade Estadual de Campinas - UNICAMP - Campinas (SP), Brasil. Departamento de Ginecologia e Obstetrícia da Faculdade de Ciências Médicas da Universidade Estadual de Campinas - UNICAMP Campinas (SP), Brasil.

2 Departamento de Anatomia Patológica da Faculdade de Ciências Médicas da Universidade Estadual de Campinas - UNICAMP Campinas (SP), Brasil.

Conflito de interesses: não há 
After multivariate regression, the only association remaining was that of high histological grade with serous tumors (adjusted OR 15.1; 95\%Cl 2.9-77.9). We observed 58 deaths from the disease. There was no difference in overall survival between women with serous carcinoma and women with non-serous carcinoma (HR 0.4; $95 \% \mathrm{Cl} 0.1-1.1)$. It was observed that women aged 50 years or less $(\mathrm{HR} 0.4 ; 95 \% \mathrm{Cl} 0.1-0.9)$ and those who were in menacne (HR 0.3; 95\% Cl 0.1-0.9) had a longer survival compared respectively to those above 50 years of age and menopaused. High histological grade (G2 and $G 3)(p<0.01$ ), stages II-IV ( $p<0.008)$ and positive cytology ( $<<0.001)$ were significantly associated with worse prognosis. CA 125 and the presence of ascites did not correlate with survival. Survival was poor when the disease was diagnosed in stages II to IV and compared to stage I (log-rank p<0.0 1 ) regardless of histological type (serous and non-serous). CONCLUSIONS: The proportion of high histological grade (G2 and G3) was significantly higher among serous than non-serous carcinomas. Serous and non-serous histological types were not related to overall survival.

\section{Introdução}

Embora o câncer de ovário não seja a neoplasia mais prevalente entre as mulheres, ele é o mais letal dos cânceres ginecológicos. Estima-se aproximadamente 200 mil casos novos a cada ano mundialmente. Nos Estados Unidos da América, é a segunda neoplasia ginecológica mais comum, sendo a quinta causa de morte por câncer em mulheres ${ }^{1}$. No Brasil, sua incidência varia conforme as diferentes regiões, sendo que em Porto Alegre e São Paulo a sua incidência supera a dos países industrializados $(11 / 100.000)^{2,3}$. Entre as neoplasias ovarianas malignas, as de origem epitelial são as mais frequentes, e são classificadas conforme o tipo celular: seroso, mucinoso, endometrióide, de células claras, de células transicionais e indiferenciados ${ }^{4,5}$.

Os carcinomas serosos são os mais comuns, correspondem a aproximadamente 80 a $85 \%$ dos carcinomas do ovário, sendo bilaterais em até $25 \%$ dos casos. Existem dois diferentes tipos de carcinomas serosos de ovário: o carcinoma seroso bem diferenciado, ou de baixo grau, eventualmente associado a áreas de tumor borderline e adenoma. Esse tumor costuma ter progressão lenta e bom prognóstico. Porém, mais frequentemente, o carcinoma seroso se apresenta com alto grau histológico, com acentuada atipia celular, arquitetura papilífera, áreas em arranjo glandular, cribriforme, microcístico, sólido ou trabecular, sendo geralmente diagnosticado em estádios avançados e com evolução desfavorável ${ }^{4,6}$. Recentemente, alguns autores têm postulado que o carcinoma seroso de ovário seria originado a partir do carcinoma seroso intraepitelial da tuba de Falópio, cujas células se implantariam no ovário. A implantação direta de células do epitélio das fímbrias da tuba para a superfície do ovário, em áreas de epitélio roto pela ovulação, formaria cistos de inclusão, com posterior transformação para carcinoma seroso de baixo ou alto grau histológico ${ }^{7,8}$.

Os outros tipos histológicos de carcinoma de ovário são mais raros. Estudos recentes demonstram que os carcinomas mucinosos do ovário são raros, correspondendo a menos de $5 \%$ dos carcinomas ${ }^{8}$. São geralmente bem diferenciados e diagnosticados em estádio inicial.
Os carcinomas endometrioides (10\%) e de células claras $(5 \%)$ também são frequentemente diagnosticados em estádios iniciais. O carcinoma de células transicionais é raro e se assemelha ao carcinoma urotelial das vias urinárias e, por definição, não deve apresentar áreas típicas de tumor de Brenner benigno?. Finalmente, são classificadas como carcinoma indiferenciado as neoplasias sem nenhuma diferenciação histológica, em arranjo sólido predominante $e^{4,10}$.

A classificação morfológica dos carcinomas de ovário não é simples e a concordância interobservador é relativamente baixa. Com o emprego de estudos moleculares foi observado que os vários tipos histológicos podem ser encarados como entidades distintas, com diferentes vias de patogênese, distinto comportamento biológico e resposta ao tratamento ${ }^{4}$. Estudos recentes sugerem uma correlação entre o tipo histológico, estádio ao diagnóstico e prognóstico e, embora as mulheres com carcinoma de ovário sejam tratadas de forma similar, a evolução da doença e sua resposta ao tratamento podem $\operatorname{variar}^{10,11}$. É muito provável que as estatísticas desfavoráveis dos pacientes com carcinoma do ovário reflitam o pouco conhecimento na origem e comportamento dessa doença.

Não existe consenso na literatura em relação ao papel do tipo histológico como fator prognóstico independente de sobrevida em mulheres com carcinoma de ovário ${ }^{12,13}$. Sabe-se que o estádio ao diagnóstico, grau histológico, volume residual do tumor após primeira cirurgia estão associados à sobrevida ${ }^{14,15}$. Existem relatos nos quais os tumores serosos mostraram prognóstico menos favorável quando comparados a outros tipos histológicos ${ }^{16}$. Assim, o objetivo deste estudo foi comparar as características clinicopatológicas dos carcinomas serosos e não serosos de ovário e identificar os fatores associados à sobrevida.

\section{Métodos}

Este é um estudo de coorte com coleta retrospectiva, para o qual foram selecionadas as mulheres submetidas à cirurgia por carcinoma de ovário no Hospital da Mulher Prof. Dr. José Aristodemo Pinotti - Centro de Atenção 
Integral a Saúde da Mulher (CAISM) da Universidade Estadual de Campinas (UNICAMP). O projeto foi aprovado pelo Comitê de Ética em Pesquisa (CEP).

Foram selecionadas as mulheres operadas no período de 1993 a 2008, e o seguimento foi avaliado até dezembro de 2010. Foram incluídos no estudo os casos em que os blocos de parafina correspondentes às lâminas da histologia estavam disponíveis e cujos prontuários estavam completos para obtenção dos dados clínicos. Todas as análises histológicas foram realizadas em material coletado antes da realização de tratamentos sistêmicos (quimioterapia). Foram identificadas 172 mulheres com diagnóstico histológico confirmado de carcinoma primário de ovário. Todas as lâminas foram coradas com hematoxilina e eosina e foram revisadas pelo mesmo patologista ginecológico, que classificou as lesões histologicamente, segundo a classificação da $\mathrm{OMS}^{10}$. Incluímos exclusivamente os casos cuja avaliação histológica permitisse a classificação precisa em um tipo histológico específico. Foram estudadas 152 mulheres nas quais o tipo histológico foi claramente estabelecido: 81 pacientes com carcinoma seroso e 71 pacientes com tumores não serosos (17 com carcinoma endometrioide, $44 \mathrm{com}$ carcinoma mucinoso e $10 \mathrm{com}$ carcinoma de células claras).

Os dados clínicos foram obtidos dos prontuários médicos: idade, estado menopausal, tipo histológico, grau histológico, estádio da doença, níveis séricos de CA125, presença de ascite, tipo de cirurgia realizada e realização de quimioterapia adjuvante. A idade foi calculada em anos completos no momento da cirurgia. $\mathrm{O}$ grau histológico foi classificado em grau 1 ou tumores bem diferenciados e graus 2 e 3 , tumores moderadamente e pouco diferenciados, respectivamente. Para análise estatística, tumores graus 2 e 3 foram agrupados como tumores de alto grau, segundo a sugestão de Ayhan et al. ${ }^{6}$. O estádio patológico foi classificado de acordo com o estádio da $\mathrm{FIGO}^{17}$. Os níveis séricos de CA 125 foram avaliados antes da cirurgia e classificados em unidades internacionais por mililitro. Foram classificados em valores menores ou maiores de $250 \mathrm{U} / \mathrm{mL}$, baseado na mediana dos valores.

A cirurgia realizada foi classificada em cirurgia de estadiamento para mulheres com doença restrita ao ovário e citorredutora em pacientes com doença avançada. A citorredução foi considerada ótima quando houve doença residual menor que um centímetro nas pacientes com doença avançada. Pacientes com doença residual maior que um centímetro e aquelas com doença não passível de ressecção foram agrupadas como citorredução subótima. A citologia foi realizada no líquido ascítico ou no lavado peritoneal. A presença de ascite foi avaliada com ultrassonografia, tomografia computadorizada e na cirurgia. A quimioterapia foi realizada de acordo com protocolo do serviço, incluindo esquemas com carboplatina associada à ciclofosfamida ou taxol.

A análise estatística foi realizada utilizando o programa $R$ Environment for Statistical Computing ( $R$ Environment $)^{18}$. A significância estatística foi estabelecida em $5 \%(\mathrm{p}=0,05)$ e os intervalos de confiança em 95\% (IC95\%). Foram inicialmente calculados os odds ratios (OR) brutos e respectivos intervalos de confiança para as características clínicas e patológicas, comparando tumores serosos e não serosos. A seguir, foi elaborado um modelo de regressão logística multivariada, para as mesmas características clinicopatológicas e derivados os $O R$ ajustados. A sobrevida total foi calculada considerando o intervalo de tempo desde o diagnóstico cirúrgico até o óbito ou último contato com a paciente. $\mathrm{O}$ estado da paciente ao último contato foi referido como vivo ou óbito segundo informações do prontuário médico. A média de seguimento deste grupo de mulheres foi de 42 meses, (mínimo-máximo 17-60 meses).

O cálculo do tamanho amostral para o modelo de sobrevida exigia uma amostra de 105 mulheres. Com isso, pudemos elaborar um modelo multivariado de Cox Proportional Hazards, a partir do qual foram calculados os Hazard Ratios (HR) com os respectivos IC95\% para as variáveis clínicas e patológicas em relação à sobrevida total. Por fim, foi elaborado um gráfico de sobrevida total, comparando mulheres nos seguintes grupamentos: a) tumores não serosos estádio $I$, b) tumores serosos estádio I, c) tumores não serosos estádios II a IV e d) tumores serosos estádios II a IV. As respectivas curvas foram comparadas pelo teste de Log-rank.

\section{Resultados}

As características clinicopatológicas das pacientes em função dos tipos histológicos dos tumores estão descritas na Tabela 1. Entre as 81 mulheres com carcinoma seroso, $57(70,3 \%)$ tinham mais que 50 anos, o que ocorria com $38(54,3 \%)$ daquelas com carcinoma não seroso (OR bruto 0,7 ; IC95\% 0,3-1,6). Sessenta e quatro mulheres com carcinoma seroso $(80 \%)$ e 42 com carcinoma não seroso $(60 \%)$ estavam na menopausa (OR bruto 0,4; IC95\% $0,2-1,0)$. Após regressão logística multivariada, não se observou diferença significativa relacionada à idade ou ao estado reprodutivo (antes ou após a menopausa). Mulheres com carcinoma seroso apresentaram prevalência significativamente maior de graus histológicos 2 e 3 ( 74 casos: $92,5 \%$ ), comparada aos não serosos ( 35 casos: 49,3\%) (OR ajustado 15,1; IC95\% 2,9-77,9). Quanto ao estádio ao diagnóstico, $66(81,5 \%)$ mulheres com carcinoma seroso apresentaram doença em estádios II a IV, comparado a $30(42,3 \%)$ mulheres com carcinoma 
Tabela 1. Distribuição das mulheres com carcinoma do ovário de tipos histológicos seroso e não seroso, segundo as características clinicopatológicas

\begin{tabular}{|c|c|c|c|c|c|c|c|c|}
\hline \multirow{3}{*}{ Características clinicopatológicas } & \multicolumn{4}{|c|}{ Tipo histológico } & \multirow{3}{*}{ OR bruto } & \multirow{3}{*}{ IC95\% } & \multirow{3}{*}{ OR ajustado } & \multirow{3}{*}{ IC95\% } \\
\hline & \multicolumn{2}{|c|}{ Seroso } & \multicolumn{2}{|c|}{ Não seroso } & & & & \\
\hline & n & $\%$ & n & $\%$ & & & & \\
\hline \multicolumn{9}{|l|}{ Idade em anos completos } \\
\hline 50 anos ou menos & 23 & 23,7 & 32 & 45,7 & 0,7 & $0,3-1,6$ & 2,2 & $0,6-8,3$ \\
\hline Ignorado & \multicolumn{2}{|c|}{1} & \multicolumn{2}{|c|}{1} & & & & \\
\hline Pós-menopausa & 64 & 80 & 42 & 60 & Referência & & Referência & \\
\hline Pré-menopausa & 16 & 20 & 28 & 40 & 0,4 & $0,2-1,0$ & 0,4 & $0,1-1,9$ \\
\hline Ignorado & \multicolumn{2}{|c|}{1} & \multicolumn{2}{|c|}{1} & & & & \\
\hline \multicolumn{9}{|l|}{ Grau histológico } \\
\hline I & 15 & 18,5 & 41 & 57,7 & Referência & & Referência & \\
\hline II-IV & 66 & 81,5 & 30 & 42,3 & 7,9 & $3,2-19,7$ & 2,7 & $0,7-10,7$ \\
\hline \multicolumn{9}{|l|}{ CA 125} \\
\hline$<250 \mathrm{U} / \mathrm{mL}$ & 31 & 40,8 & 41 & 67,2 & Referência & & Referência & \\
\hline$>250 \mathrm{U} / \mathrm{mL}$ & 45 & 59,2 & 20 & 32,8 & 3,8 & $1,7-8,7$ & 1,2 & $0,4-4,0$ \\
\hline Ignorado & \multicolumn{2}{|c|}{5} & \multicolumn{2}{|c|}{10} & & & & \\
\hline Media( $\pm D P)$ & 2377,37 & \pm 5695 & 747,47 & \pm 2470 & & & & \\
\hline \multicolumn{9}{|l|}{ Citologia } \\
\hline Negativa & 34 & 56,7 & 46 & 75,4 & Referência & & Referência & \\
\hline Positiva & 26 & 43,3 & 15 & 24,6 & 8,7 & $3,2-23,9$ & 1,1 & $0,3-3,7$ \\
\hline Estádio I & 15 & 18,5 & 41 & 57,7 & Referência & & & \\
\hline Citorredução ótima & 23 & 28,4 & 12 & 16,9 & 6,9 & $2,2-21,4$ & 0,6 & $0,2-2,5$ \\
\hline Citorredução subótima & 43 & 53,1 & 18 & 25,4 & NC & NC & NC & NC \\
\hline Quimioterapia & & & & & & & & \\
\hline Estádio I & 15 & 18,5 & 41 & 57,7 & Referência & & & \\
\hline Estádio II-IV-Não & 7 & 8,6 & 3 & 4,2 & 3,8 & $1,7-8,7$ & 1,6 & $0,2-17,2$ \\
\hline Estádio II-IV-Sim & 59 & 72,8 & 27 & 38,1 & NC & NC & NC & NC \\
\hline Total & 81 & 53,3 & 71 & 46,7 & & & & \\
\hline
\end{tabular}

DP: desvio padrão; OR:odds ratio; IC95\%: intervalo de confiança a 95\%; NC: não calculável.

não seroso (OR bruto 7,9; IC95\% 3,2-19,7). Na análise univariada, o nível sérico médio de CA125 foi significativamente maior em mulheres com carcinoma seroso do que nos não serosos (OR bruto 3,8; IC95\% 1,7-8,7). A citologia foi positiva para células neoplásicas em 26 $(43,3 \%)$ mulheres com carcinoma seroso e em apenas 15 $(24,6 \%)$ mulheres com carcinoma não seroso, e a ascite foi encontrada em $40(53,3 \%)$ mulheres com carcinoma seroso e em apenas $23(34,3 \%)$ pacientes com carcinoma não seroso. Em relação à cirurgia de citorredução, 23 mulheres com carcinoma seroso avançado $(28,4 \%)$ e 12 mulheres com carcinoma não seroso avançado $(16,9 \%)$ tiveram cirurgia considerada ótima. Mais da metade das mulheres com carcinoma seroso foram submetidas a citorredução subótima. A análise da Tabela 1 demonstra que o carcinoma seroso apresenta características clinicopatológicas diferentes dos não serosos, representadas por alto grau histológico, maior estádio da doença ao 
diagnóstico, maior nível de CA125, frequência maior de citologia positiva do líquido peritonial.

Foram observados 58 óbitos pela doença e na Tabela 2, foi avaliada associação das características clinicopatológicas

Tabela 2. Avaliação da sobrevida total segundo as características clinicopatológicas das mulheres com carcinoma de ovário, após regressão múltipla

\begin{tabular}{|c|c|c|c|}
\hline & Hazard Ratios & IC95\% & Valor p ajustado \\
\hline \multicolumn{4}{|l|}{ Tipo histológico } \\
\hline Seroso & Referência & & \\
\hline Não seroso & 0,4 & $0,1-1,1$ & 0,07 \\
\hline \multicolumn{4}{|l|}{ Idade } \\
\hline Mais que 50 anos & Referência & & \\
\hline 50 anos ou menos & 0,4 & $0,1-0,9$ & 0,04 \\
\hline \multicolumn{4}{|l|}{ Estado menopausal } \\
\hline Pós-menopausa & Referência & & \\
\hline Pré-menopausa & 0,3 & $0,1-0,9$ & 0,02 \\
\hline \multicolumn{4}{|l|}{ Grau Histológico } \\
\hline 1 & Referência & & \\
\hline $2-3$ & 8,4 & $1,7-43$ & 0,01 \\
\hline \multicolumn{4}{|l|}{ Estádio } \\
\hline I & Referência & & \\
\hline II-IV & 10,5 & $1,8-60$ & 0,008 \\
\hline \multicolumn{4}{|l|}{ CA 125} \\
\hline$<250 \mathrm{U} / \mathrm{mL}$ & Referência & & \\
\hline$>250 \mathrm{U} / \mathrm{mL}$ & 1,4 & $0,3-2,1$ & 0,66 \\
\hline \multicolumn{4}{|l|}{ Citologia } \\
\hline Negativo & Referência & & \\
\hline Positivo & 5,0 & $1,8-13,6$ & 0,001 \\
\hline \multicolumn{4}{|l|}{ Ascite } \\
\hline Não & Referência & & \\
\hline Sim & 1,0 & 0,2 a 1,6 & 0,34 \\
\hline \multicolumn{4}{|l|}{ Cirurgia } \\
\hline Estádio I & Referência & & \\
\hline Citoredução ótima & 1,0 & 0,1 a 1,1 & 0,07 \\
\hline Citoredução subótima & $\mathrm{NC}$ & $\mathrm{NC}$ & $\mathrm{NC}$ \\
\hline
\end{tabular}

IC95\%: intervalo de confiança a 95\%; NC:não calculável.

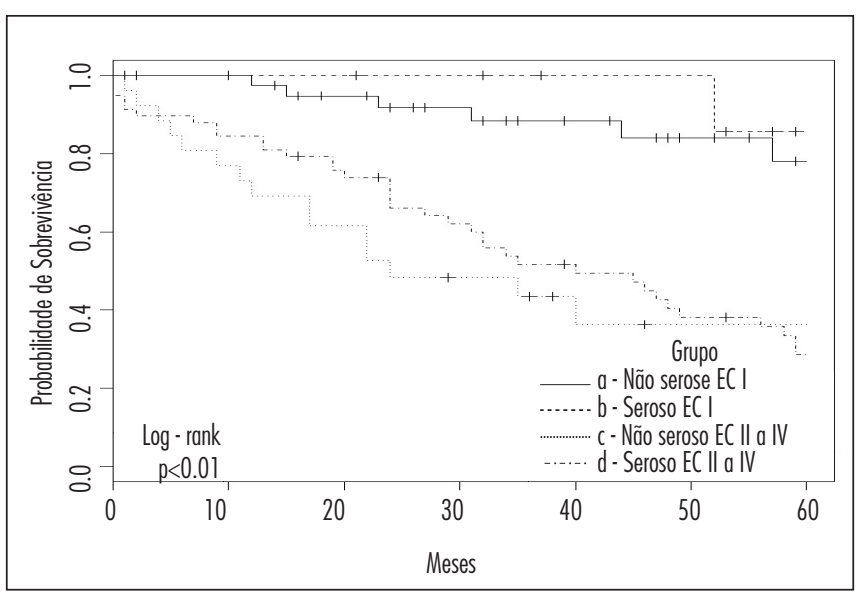

Figura 1. Sobrevida Total comparada com teste de log-rank por tipo histológico (seroso versus não seroso) e estádio (I versus II a IV), $p<0,01$ comparando estádio I versus estádio II-IV, independentemente do tipo histológico. com a sobrevida total. Para isso foi realizada a análise multivariada, que demonstrou que na sobrevida total não houve diferença significativa entre as mulheres com carcinoma seroso quando comparadas com carcinoma não seroso (HR 0,4; IC95\% 0,1-1,1; p=0,07). Observou-se que mulheres com 50 anos ou menos (HR 0,4; IC95\% $0,1-0,9 ; \mathrm{p}=0,04)$, e aquelas que estavam na menacme (HR 0,3; IC95\% 0,1-0,9; $\mathrm{p}=0,02$ ) apresentaram maior sobrevida em comparação, respectivamente, àquelas acima de 50 anos e na menopausa. Tumores de graus 2 e $3(\mathrm{p}<0,01)$, doença nos estádios II-IV $(\mathrm{p}<0,008)$, e citologia positiva $(\mathrm{p}<0,001)$ estiveram significativamente associados com pior sobrevida. Não foi possível avaliar o impacto da cirurgia e quimioterapia na análise multivariada, devido à grande proporção de tumores em estádios iniciais.

Comparando as mulheres com tumores serosos e não serosos, estádios I e II a IV pelo teste de Log-rank, observamos que houve pior sobrevida quando a doença foi diagnosticada nos estádios II a IV quando comparadas com aquelas diagnosticadas no estádio I, independentemente do tipo histológico (Log-rank p <0,01) (Figura 1).

\section{Discussão}

Neste estudo, em que comparamos mulheres com carcinomas de ovário agrupados em tipos serosos e não serosos, observamos que as características clinicopatológicas dos dois grupos são diferentes, demonstrando que o carcinoma seroso tem grau histológico mais alto, apresenta-se em estádio mais avançado ao diagnóstico, ocorre principalmente na pós-menopausa, está associado ao maior nível do CA125 e à maior frequência de citologia peritonial positiva. Esses dados estão de acordo com trabalhos recentes da literatura ${ }^{4,6,8}$, que apontam para diferentes vias de patogênese para os carcinomas serosos de alto grau do ovário em relação aos outros tipos histológicos. Segundo Kurman e Shih ${ }^{8}$, existem duas vias principais de carcinogênese ovariana: um grupo de tumores, denominados tipo I, que inclui essencialmente carcinomas serosos e endometrioides bem diferenciados, carcinoma de células claras e transicionais, que evoluem de forma mais indolente, são geralmente detectados ainda restritos aos ovários e são geneticamente estáveis. Apresentam-se frequentemente associados a áreas benignas e borderline. Já os tumores do tipo II são mais agressivos, com acentuada atipia celular, de alto grau histológico, quase sempre diagnosticados em estádios avançados. Os tumores do tipo II são representados pelos carcinomas serosos de alto grau histológico (graus 2 ou 3), carcinoma indiferenciado, carcinossarcoma ou tumor mesodérmico misto 
maligno e não se reconhecem lesões precursoras neste $\operatorname{grupo}^{5,8,10,19}$.

Quando avaliamos o tempo de sobrevida total pela análise multivariada, não identificamos diferenças significativas entre os tipos histológicos seroso e não seroso. Entretanto, idade acima de 50 anos, menopausa, tumores de graus histológicos 2 ou 3, estádio II a IV e com citologia peritoneal positiva foram fatores significativos de pior prognóstico.

Em muitos estudos recentes, o grau histológico apresenta-se como fator prognóstico independente relacionado à sobrevida de mulheres com carcinoma de ovário. Por muitos anos os tumores de ovário foram classificados em três graus de diferenciação histológica: bem diferenciados, moderadamente e pouco diferenciados. Recentemente, Ayhan et al. ${ }^{6}$ demonstraram que o perfil genético e o comportamento biológico do carcinoma seroso grau 2 é essencialmente o mesmo do carcinoma seroso grau histológico 3, achado que sustenta a pertinência de um sistema de classificação em apenas duas categorias: baixo grau e alto grau. Assim, optamos por analisar todos os casos seguindo esta nova classificação, agrupando os tumores serosos de graus histológicos 2 e 3 em apenas um grupo, denominado alto grau. Chan et al. ${ }^{20}$ observaram que em mulheres com carcinoma de ovário estádio I, o grau histológico esteve associado tanto com o tempo livre de doença quanto com a sobrevida total. Na nossa casuística, apenas $10 \%$ das mulheres com carcinomas serosos apresentaram tumores bem diferenciados.

No presente estudo, mulheres com 50 anos ou menos e na pré-menopausa apresentaram sobrevida total significativamente maior que aquelas com mais de 50 anos e na pós-menopausa. Esse dado está em concordância com a literatura. Vários estudos avaliaram a idade como fator prognóstico em mulheres com carcinoma de ovário: alguns autores observaram que mulheres mais jovens apresentam melhor prognóstico, em todos os estágios, quando comparadas às mulheres mais velhas ${ }^{14,19,21,22}$. Chan et al. ${ }^{22}$, em um estudo incluindo 28.165 mulheres, observaram que mulheres com menos de 60 anos apresentavam sobrevida significativamente maior mesmo após ajuste pelo grau histológico e estádio do tumor. Os autores, porém, acrescentam que mulheres muito jovens (até 30 anos) tendem a apresentar mais frequentemente tumores bem diferenciados detectados em estádios inicias, e que o bom prognóstico estaria, portanto, associado ao comportamento indolente desses tumores. Entre as mulheres com idade entre 30 e 60 anos, Chan et al. ${ }^{22}$ observaram que $40 \%$ tiveram a doença diagnosticada em estádio I, comparado com apenas $25 \%$ das mulheres com mais de 60 anos.
Os carcinomas de ovário detectados em estádios iniciais apresentam melhor prognóstico quando comparados àqueles detectados em estádios mais avançados, em todos os estudos ${ }^{19,23}$. Em pacientes com doença avançada, estádio II a IV, a presença de doença residual após a primeira cirurgia é um fator prognóstico importante ${ }^{14}$. Da mesma forma, a presença de células neoplásicas no lavado peritoneal ou na ascite foi associada à menor sobrevida. Entretanto, a presença de ascite por si não se apresentou como fator prognóstico independente, assim como em outros estudos ${ }^{19}$. A sobrevida de mulheres com carcinoma de ovário é negativamente afetada pelo estádio da doença no momento em que o diagnóstico é realizado. Em apenas $25 \%$ das pacientes o diagnóstico é feito em estádio inicial, quando a sobrevida em cinco anos é de $80 \%$. Devido à carência de sinais e sintomas específicos, os carcinomas de ovário são diagnosticados em estádios avançados (III e IV) na maioria das pacientes $(70 \%)$, e a sobrevida média livre de doença não ultrapassa 18 meses. Menos da metade das mulheres ( $45 \%$ ) sobrevive por mais de cinco anos após o diagnóstico ${ }^{17,24-26}$.

O presente estudo apresenta alguns aspectos limitantes: por ser retrospectivo, não pudemos uniformizar o tratamento, sendo que foram utilizados vários esquemas de quimioterapia. Por incluirmos casos em estádio I, não foi possível avaliar o impacto da citorredução na sobrevida na análise multivariada. Por outro lado, este estudo se destaca por apresentar uma casuística suficiente de carcinoma de ovário tratadas e seguidas num único serviço.

Em conclusão, embora mulheres com carcinoma seroso sejam mais frequentemente menopausadas, com doença em estádio avançado, CA125 elevado e citologia peritoneal positiva, essas variáveis não se mantiveram significativas quando ajustadas pelo grau de diferenciação histológica. A proporção de carcinomas moderadamente e pouco diferenciados foi significativamente maior entre mulheres com carcinomas serosos comparados com não serosos. Da mesma forma, quando ajustados por todos os fatores incluídos na análise multivariada, o tipo histológico (seroso ou não seroso) não esteve associado à sobrevida global. A idade mais avançada, menopausa, grau histológico 2 e 3, estádio II a IV e citologia peritoneal positiva para células neoplásicas estiveram significativamente relacionados com pior sobrevida total, independentemente do tipo histológico.

\section{Agradecimentos}

À Fundação de Amparo à Pesquisa do Estado de São Paulo, processo no 2009/17097-1; à ex-aluna de iniciação científica Patrícia Ferreira, bolsista no período de 12 meses (2009/18306-3). 
1. American Cancer Society [Internet]. Cancer facts and figures 2008 [cited 2012 Jan 3]. Available from: http://www.cancer.org/acs/ groups/content/@nho/documents/document/2008cafffinalsecu redpdf.pdf

2. Jemal A, Siegel R, Ward E, Hao Y, Xu J, Murray T, et al. Cancer statistics, 2008. CA Cancer J Clin. 2008;58(2):71-96.

3. Brasil. Ministério da Saúde. Instituto Nacional do Câncer (INCA). Coordenação Nacional de Prevenção e Vigilância do Câncer [Internet]. Câncer no Brasil: dados dos registros de base populacional. Rio de Janeiro; 2003 [cited 2012 Jan 3]. Available from: <http:// wwwl inca.gov.br/regpop/2003/index.asp?link=conteudo_view. asp\&ID $=10$

4. Soslow RA. Histologic subtypes of ovarian carcinoma: an overview. Int J Gynecol Pathol. 2008;27(2):161-74.

5. Bell DA. Origins and molecular pathology of ovarian cancer. Mod Pathol. 2005; 18(Suppl 2)S19-32.

6. Ayhan A, Kurman RJ, Yemelyanova A, Vang R, Logani S, Seidman $J D$, et al. Defining the cut point between low-grade and high-grade ovarian serous carcinomas: a clinicopathologic and molecular genetic analysis. Am J Surg Pathol. 2009;33(8):1220-4.

7. Diniz PM, Carvalho JP, Baracat EC, Carvalho FM. Fallopian tube origin of supposed ovarian high-grade serous carcinomas. Clinics. $2011 ; 66(1): 73-6$.

8. Kurman RJ, Shih IM. The origin and pathogenesis of epithelial ovarian cancer: a proposed unifying theory. Am J Surg Pathol. 2010;34(3):433-43.

9. Tazi EM, Lalya I, Tazi MF, Ahellal Y, M'rabti H, Errihani $H$. Transitional cell carcinoma of the ovary: a rare case and review of literature. World J Surg Oncol. 2010;14;8:98.

10. McCluggage WG. Morphological subtypes of ovarian carcinoma: a review with emphasis on new developments and pathogenesis. Pathology. 2011 ; 43(5):420-32.

11. Farley J, Ozbun LL, Birrer M. Genomic analysis of epithelial ovarian cancer. Cell Res. 2008; 18(5):538-48.

12. Brun JL, Feyler A, Chêne G, Saurel J, Brun G, Hocké C. Long-term results and prognostic factors in patients with epithelial ovarian cancer. Gynecol Oncol. 2000;78(1):21-7.

13. Hosono S, Kajiyama H, Mizuno K, Sakakibara K, Matsuzawa $K$, Takeda A, et al. Comparison between serous and non-serous ovarian cancer as a prognostic factor in advanced epithelial ovarian carcinoma after primary debulking surgery. Int J Clin Oncol. $2011 ; 16(5): 524-32$.

14. Winter WE 3rd, Maxwell GL, Tian C, Carlson JW, Ozols RF, Rose PG, et al. Prognostic factors for stage III epithelial ovarian cancer: a Gynecologic Oncology Group Study. J Clin Oncol. 2007;25(24):3621-7.

15. Brun JL, Bouzigon E, Saurel J, Chêne G, Briex M, Brun G, et al. Prognostic factors for survival of ovarian epithelial cancers: a propos of 287 cases. Gynecol Obstet Fertil. 2000;28(3):22331.

16. Makar AP, Baekelandt M, Tropé CG, Kristensen GB. The prognostic significance of residual disease, FIGO substage, tumor histology, and grade in patients with FIGO stage III ovarian cancer. Gynecol Oncol. 1995;56(2):175-80.

17. Benedet JL, Bender H, Jones H 3rd, Ngan HY, Pecorelli S. FIGO staging classifications and clinical practice guidelines in the management of gynecologic cancers. FIGO Committee on Gynecologic Oncology. Int J Gynaecol Obstet. 2000;70(2):209-62.

18. R Development Core Team. R: a language and environment for statistical computing [Internet]. Vienna: R Foundation for Statistical Computing; 2004 [cited 2011 Apr 5]. Available from: http://Rproject.org

19. Canevari S, Gariboldi M, Reid JF, Bongarzone I, Pierotti MA. Molecular predictors of response and outcome in ovarian cancer. Crit Rev Oncol Hematol. 2006;60(1):19-37.

20. Chan JK, Tian C, Monk BJ, Herzog T, Kapp DS, Bell J, et al. Prognostic factors for high-risk early-stage epithelial ovarian cancer: a Gynecologic Oncology Group Study. Cancer. 2008; $112(10): 2202-10$.

21. Chan JK, Loizzi V, Lin YG, Osann K, Brewster WR, DiSaia PJ. Stages III and IV invasive ephitelial ovarian carcinoma in younger versus older women: what prognostic factors are important? Obstet Gynecol. 2003;102(1):156-61.

22. Chan JK, Urban R, Cheung MK, Osann K, Shin JY, Husain A, et al. Ovarian cancer in younger vs older women: a population-based analysis. Br J Cancer. 2006;95(10):1314-20.

23. Vergote I, De Brabanter J, Fyles A, Bertelsen K, Einhorn N, Sevelda $P$, et al. Prognostic importance of degree of differentiation and cyst rupture in stage I invasive epithelial ovarian carcinoma. Lancet. $2001 ; 357(9251): 176-82$.

24. Weir HK, Thun M, Hankey BF, Ries LA, Howe HL, Wingo PA, et al. Annual report to the nation on the status of cancer, 1975-2000, featuring the uses of surveillance data for cancer prevention and control. J Natl Cancer Inst. 2003;95(17):1276-99.

25. Aletti GD, Gallenberg MM, Cliby WA, Jatoi A, Hartmann LC. Current management strategies for ovarian cancer. Mayo Clin Proc. 2007;82(6):751-70.

26. Permuth-Wey J, Sellers TA. Epidemiology of ovarian cancer. Methods Mol Biol. 2009;472:413-37. 